\title{
Intelligent Urban Mobility: Pedestrian and Bicycle Seamless Navigation
}

\author{
Estefania Munoz Diaz and Fabian de Ponte Müller and Eduardo Pérez González \\ German Aerospace Center (DLR) \\ Institute of Communications and Navigation \\ Oberpfaffenhofen, 82234 Wessling, Germany \\ Email: Estefania.Munoz@dlr.de
}

\begin{abstract}
Mobility is evolving in urban scenarios and multimodality is the key to a more efficient transportation. In this article we propose a multimodal intelligent navigation system for urban indoor and outdoor environments. Our method is based on wearable sensors mounted on different locations on the human body. The algorithm based on a loose INS/GNSS fusion with magnetometers switches intelligently and seamlessly between the transportation modes walking and riding a bicycle. We also propose an algorithm to cover the still unsolved issue regarding coasting and braking periods for bicycle navigation in GNSS denied scenarios. We have performed an extensive measurement campaign of more than $12 \mathrm{~km}$ to test the performance of the proposed algorithms and we have used a Precise Point Positioning solution as ground truth to compute the error. We prove that our method is able to successfully estimate the forward speed of the bicycle during coasting or braking periods. Likewise, we prove that our navigation system switches seamlessly between walking and riding a bicycle and is also able to bridge short GNSS outages.
\end{abstract}

Index Terms-Magnetometer, coasting, transition, outdoors, indoors, multimodal transportation, ubiquitous navigation.

\section{INTRODUCTION}

According to the European Commission, 123,000 people were seriously injured and 25,000 people were killed in 2016 in Europe by traffic accidents. Almost $29 \%$ of the fatalities occurred among non-motorized road users which are called Vulnerable Road Users (VRUs).

Traditionally, the approach to protect VRUs has been relying on detection sensors like cameras, radars and laser-scanners mounted on the vehicles and/or embedded in the road infrastructure, e.g. lamp posts [1], [2]. The on-board sensors in the vehicle detect the presence of pedestrians and bicycles and then either warn the driver or actuate directly on the car by braking or performing an evasive manoeuver.

The traditional approach assumes a VRU devoid of any communication device, the so-called non-cooperative approach. Nowadays, however, the use of smartphones is widespread. These devices provide not only communication capabilities, but also location possibilities thanks to their many embedded sensors, e.g. inertial sensors, barometer, magnetometer and Global Navigation Satellite System (GNSS) receiver.

Therefore, a new era of VRU protection using a cooperative approach is now open. In the cooperative approach, every

978-1-5386-5635-8/18/\$31.00 (C) 2018 IEEE

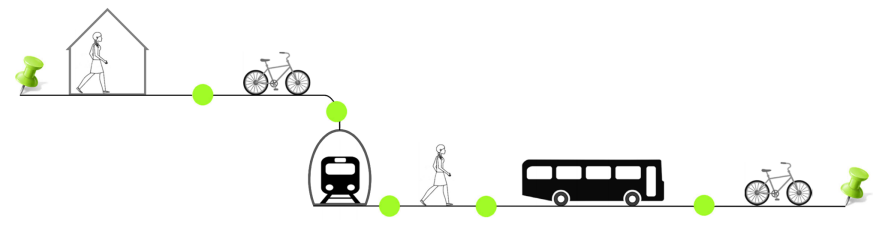

Fig. 1. Example of urban multimodal transportation to bridge origin and destination.

road user knows where she is and transmits her position periodically. There are two possibilities: either the VRUs transmit their position to the motorized vehicles, so they can run a collision detection algorithm and act accordingly; or all road users transmit their position to the cloud, where the possibility of a potential collision is calculated and the involved road users are warned.

Not only the accurate position of the VRUs is needed, but also the mean of transport they use, i.e. walking or riding a bicycle. We envision for the city of the future a clear trend on ubiquitous multimodal navigation, as represented in Figure 1. That means, in order to bridge two points, some sections will be covered walking, indoors and outdoors, and some others by public transport or riding a bicycle, among others.

Therefore, in this work we propose an intelligent urban navigation system for cycling and walking in indoor and outdoor scenarios. Additionally, we propose a novel algorithm to compute the forward speed of the bicycle during coasting and braking periods with non-vehicle-mounted sensors.

Due to the multimodality, our philosophy is to use sensors embedded in smart watches, smart glasses, smart clothes and also smartphones, rather than using vehicle-mounted sensors. We propose a multi-sensor fusion approach based on inertial sensors (INS), magnetometers and GNSS.

The state-of-the-art regarding pedestrian and bicycle navigation is summarized in Section II. Section III comprises a novel algorithm to estimate the forward speed of the bicycle while coasting and braking periods, as well as the proposed multimodal intelligent urban navigation system. The experimental results are shown in Section IV, where the measurement campaign set up is also detailed. Finally, the conclusions and outlook are referred to Section V. 


\section{State of The ART}

\section{A. Pedestrian Navigation}

Pedestrian dead-reckoning (PDR) navigation systems can make use of the inertial sensors embedded in any wearable such as smart watches, smart glasses and smart clothing. The step\&heading algorithm can always be used independent of the body location where the sensors are mounted or embedded [3]-[7]. The reader is referred to [8] for more information on the step\&heading algorithm.

The step\&heading algorithm used in this article is represented in the block diagram of Figure 2.

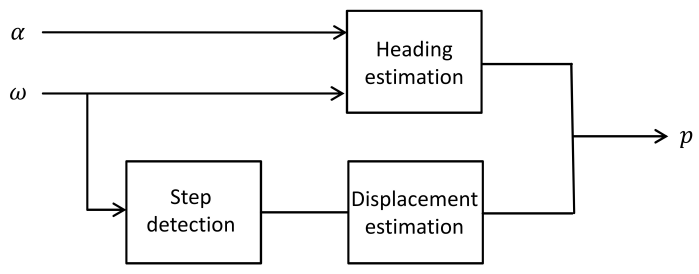

Fig. 2. Block diagram of the step\&heading algorithm.

The step\&heading algorithm is based on the following equations:

$$
\begin{aligned}
& p_{\mathrm{x}}^{k}=p_{\mathrm{x}}^{k-1}+d_{\mathrm{h}}^{k} \cdot \cos \left(\psi^{k}\right), \\
& p_{\mathrm{y}}^{k}=p_{\mathrm{y}}^{k-1}+d_{\mathrm{h}}^{k} \cdot \sin \left(\psi^{k}\right),
\end{aligned}
$$

where $p_{\mathrm{x}}^{k}$ and $p_{\mathrm{y}}^{k}$ represent the position in the $\mathrm{x}$ - and $\mathrm{y}$-axis at the time $k, d_{\mathrm{h}}^{k}$ stands for the horizontal displacement, i.e. step length, at the time $k$ and $\psi^{k}$ is the heading of the pedestrian at the time $k$. Therefore, in order to compute the position of the pedestrian, two steps are necessary: the orientation estimation, to obtain the heading angle, and the displacement estimation.

The step\&heading algorithm is usually defined in 2D, as indicated in Equation (1). However, for particular sensor locations it is possible to solve 3D positioning. The authors in [9] demonstrate that, if the inertial sensors are attached to the lower limb of the pedestrian, it is possible to differentiate between walking horizontally and climbing stairs by means of the orientation of the leg of the pedestrian. The information on the walking surface allows deriving the vertical displacement $d_{\mathrm{v}}^{k}$, where $d_{\mathrm{v}}^{k}$ represents the vertical displacement from the time $k-1$ to the time $k$. In that case the position in the $\mathrm{z}$-axis is as follows:

$$
p_{\mathrm{z}}^{k}=p_{\mathrm{z}}^{k-1}+d_{\mathrm{v}}^{k} .
$$

The displacement estimation, i.e. the step length and the vertical displacement, is triggered every time a new step is detected. The step detection as well as the displacement estimation are derived using the pitch angle estimation, as detailed in [9], [10].

The heading angle as well as the pitch angle are estimated by the orientation estimator. The estimation of the orientation using only inertial sensors is explained in detail in [8]. Additionally, PDR navigation systems are usually complemented with GNSS. The heading angle extracted from the GNSS measurements $\psi_{G N S S}$ is applied as an update in the orientation estimation filter.

\section{B. Bicycle Navigation}

Cyclist dead-reckoning (CDR) navigation systems [11], [12] can also make use of the inertial sensors embedded in any wearable such as smart watches, smart glasses and smart clothing. The algorithm used namely speed\&heading is represented in the block diagram of Figure 3 for a fixed gear:

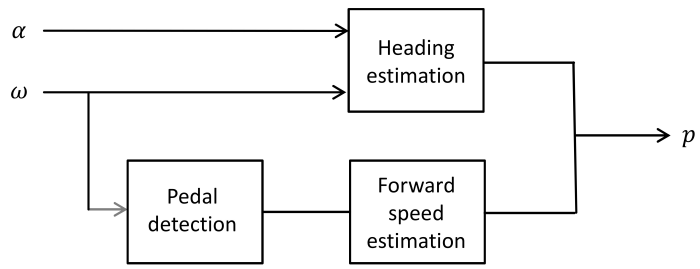

Fig. 3. Block diagram of the speed\&heading algorithm for a fixed gear.

The speed\&heading algorithm is based on the following equations (assuming a bidimensional movement model):

$$
\begin{gathered}
p_{\mathrm{x}}^{k}=p_{\mathrm{x}}^{k-1}+s^{k} \cdot \Delta t \cdot \cos \left(\psi^{k}\right), \\
p_{\mathrm{y}}^{k}=p_{\mathrm{y}}^{k-1}+s^{k} \cdot \Delta t \cdot \sin \left(\psi^{k}\right),
\end{gathered}
$$

where $p_{\mathrm{x}}^{k}$ and $p_{\mathrm{y}}^{k}$ represent the position in the $\mathrm{x}$ - and $\mathrm{y}$-axis at the time $k, \Delta t$ is the time elapsed between $k-1$ and $k$, and $\psi^{k}$ is the heading of the bicycle at the time $k$. The parameter $s^{k}$ stands for the forward speed of the bicycle at the time $k$, which is equal to $\left(2 \pi \cdot r \cdot f_{w}^{k}\right)$, being $r$ the radius of the wheel and $f_{w}^{k}$ the instantaneous wheel frequency.

Therefore, in order to compute the position of the cyclist given the radius of the wheel of the bicycle, two steps are necessary: the orientation estimation, to have the heading angle, and the wheel frequency to have the forward speed of the bicycle.

Inertial sensors are usually combined with GNSS also for bicycle navigation. For the orientation estimation, the heading angle is therefore extracted from the GNSS measurements $\psi_{G N S S}$ and applied in the navigation filter as an update.

If the user is pedaling, the frequency of the wheel $f_{w}^{k}$ is related to the pedaling frequency $f_{p}^{k}$ through the gear $g^{k}$. The CDR algorithm computes the gear when GNSS signals are available and assumes no changes during GNSS outages. When more than four satellite signals are tracked and a position-velocity-time (PVT) solution can be computed, $f_{w}^{k}$ is derived through the forward speed extracted from the GNSS $s_{\mathrm{GNSS}}$ as follows:

$$
f_{w}^{k}=\frac{s_{\mathrm{GNSS}}^{k}}{2 \pi \cdot r} .
$$

The pedaling frequency $f_{p}^{k}$ is obtained either using measurements from accelerometers or gyroscopes [11] that monitor the movement of the lower limb of the cyclist.

The values of $f_{w}^{k}$ and $f_{p}^{k}$ during GNSS fixes are used to find the gear $g^{k}$ through the predefined curves for each bicycle, 
as shown in Figure 4. Every line in the figure represents a different gear.

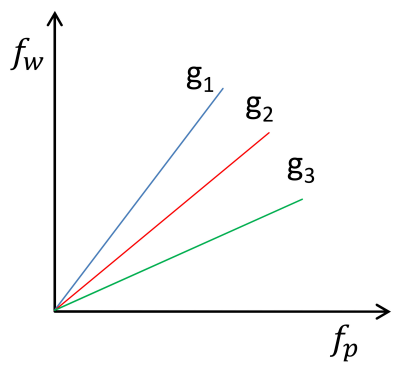

Fig. 4. The gear relates during the ride the pedaling frequency $f_{p}$ and the wheel frequency $f_{w}$.

The gear and the pedaling frequency will be further used to estimate the frequency of the wheel (Figure 4) over time in the absence of GNSS measurements.

The forward speed from the GNSS measurements $s_{\mathrm{GNSS}}^{k}$, as well as the GNSS position are also used to update the estimations of the parameters of Equation (3).

Apart from pedaling, there are other actions performed when riding a bicycle, such as coasting and braking. Generally speaking for any kind of vehicle, coasting is performing a natural deceleration of a motor when the power is removed. When riding a bicycle, coasting refers to keep moving forward without pedaling. This happens usually when riding down a hill or at corners to slightly decrease the speed, among others.

When coasting, the bicycle keeps moving forward and the pedaling frequency is zero. The same happens when braking. Coasting and braking periods are still an open issue for CDR navigation systems, since using the aforementioned method (see Figure 4), no pedaling implies a frequency of the wheel equal to zero. Without vehicle-mounted sensors, coasting and braking periods can only be solved so far using GNSS.

We propose in this article a novel algorithm to compute the forward speed of the bicycle using only magnetometers embedded in the cyclist's shoe.

\section{Proposed Method}

The proposed algorithm aims at achieving an intelligent urban mobility based on ubiquitous and multimodal navigation. Unlike the vast majority of the literature that is based on vehicle-mounted sensors, we use only body-mounted sensors to navigate in GNSS denied scenarios covering also coasting periods. Our multi-sensor approach is based on the loose fusion INS/GNSS and magnetometers.

\section{A. Coasting for Bicycle Navigation}

In this article we propose an algorithm (patent filed at the German Patent Office) to compute the frequency of the wheel for GNSS denied scenarios, such as urban, without using vehicle-mounted devices.

In our approach, the pedaling and the coasting periods are detected through the amplitude of the pitch angle estimation. The pedaling frequency $f_{p}^{k}$ is obtained through inertial sensors mounted or embedded in clothes of the lower limb of the cyclist. Figure 5(a) shows the pitch angle estimated when cycling for a sensor introduced in the front pocket of the trousers of the user. The pedaling frequency is the inverse of the time elapsed between pedal turns. The local maxima of the pitch angle estimation corresponds to the highest position of the pedal on the side where the sensor is mounted as shown in Figure 5(b).

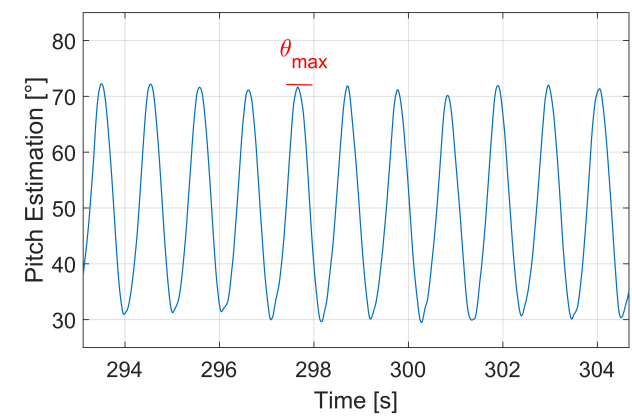

(a) Pitch angle estimation

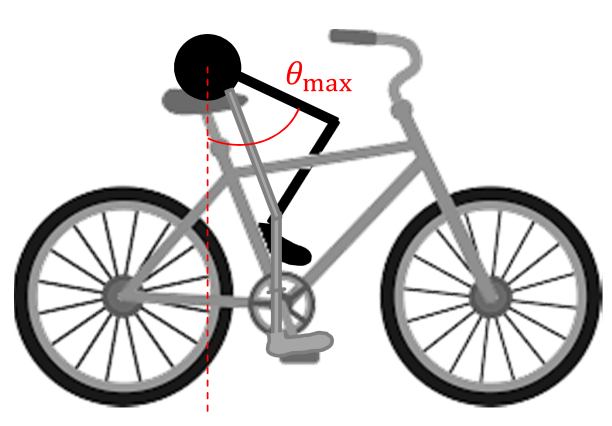

(b) Movement of the legs cycling

Fig. 5. Pitch angle estimation when riding a bicycle for the sensors introduced in the front pocket of the trousers and schema of the movement of the legs while cycling.

Figure 6 shows the pitch angle estimation resulting when the user alternates between pedaling and coasting periods. Coasting is highlighted in red.

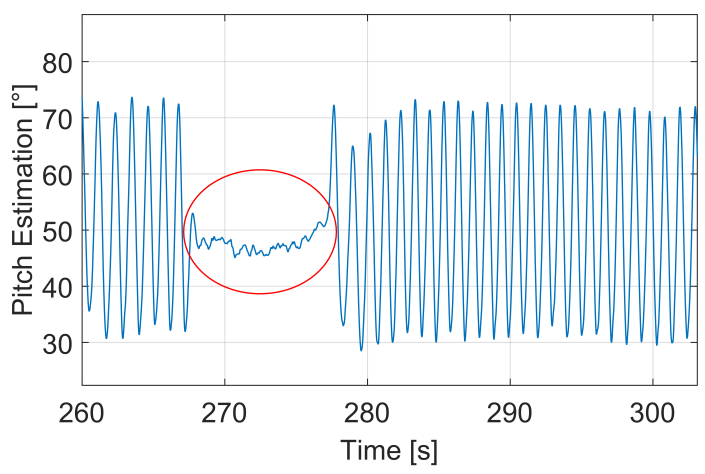

Fig. 6. Pitch angle estimation during pedaling and coasting periods. Coasting is highlighted in red.

Both, pedaling and coasting periods, are detected using 
the pitch angle estimation, which is constantly stored in a FIFO buffer of $1 \mathrm{~s}$ length. If the value of the variance of the measurements stored in the buffer does not exceed an empirically-determined threshold, it is considered that the user's leg does not move. This indication is consequently used to detect coasting periods.

The proposed algorithm to estimate the frequency of the wheel while coasting and braking requires one magnetometer embedded in the shoe. We prove that the wheels' rotation can be detected using magnetometers thanks to the metallic parts of the bicycle, such as rim, hub, spokes and gears.

When the bicycle wheel rotates, these metallic parts cause variations in the measurements of the Earth magnetic field. In our case we analyze the variations of the norm of three mutually orthogonal magnetometers. The frequency of these variations can be computed using the Fourier transform (FFT).

Figure 7 shows the measurements of the magnetometer in frequency domain. This experiment has been performed placing the bicycle upside down and moving manually only the front wheel. The sensor embedding three mutually orthogonal magnetometers has been placed approximately $3 \mathrm{~cm}$ away from the front wheel.

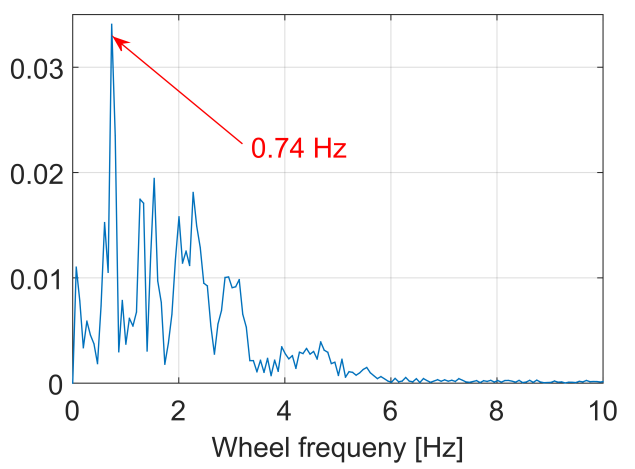

Fig. 7. The figure shows the magnetometers' measurements in frequency domain caused by the metallic parts of the bicycle.

Therefore, the highest peak of the FFT of the norm of the three mutually orthogonal magnetometers when the wheel moves at constant angular velocity, represents directly the frequency of the wheel. This can be applied during coasting or braking periods to deduce the forward speed without using GNSS or vehicle-mounted sensors, such odometers.

\section{B. Multimodal Intelligent Urban Navigation}

In order to achieve ubiquitous navigation, the proposed system integrates a multisensor approach based on pocketmounted inertial sensors, GNSS and shoe-mounted magnetometers. Additionally, the seamless detection of different means of transportation, in this case walking and cycling, is integrated in our approach.

We propose a cascaded Kalman filter, as shown in Figure 8, in which the orientation is computed first, and then the position and forward speed of the user.

For this paper, a loose INS/GNSS fusion is foreseen. The GNSS pseudoranges are processed by a proprietary software

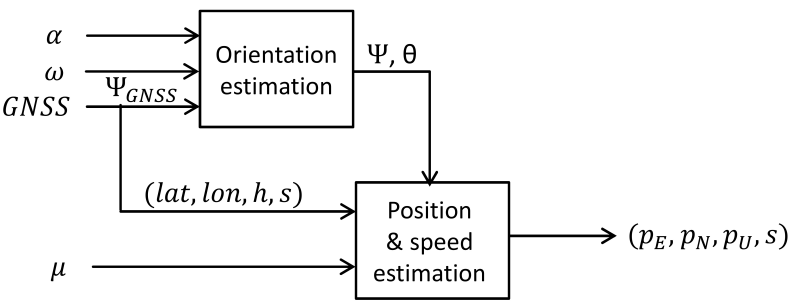

Fig. 8. Cascaded filter approach used for ubiquitous and multimodal navigation.

and the latitude, longitude, height and velocity are generated as output. Then, we decompose the velocity vector into forward speed and heading angle and use these parameters to update our proposed cascade filter.

The orientation is computed in our approach the same way independent of the mean of transportation, i.e. walking or cycling. The orientation estimation filter uses inertial measurements from the pocket-mounted sensor for the prediction stage as explained in [8]. Apart from the updates mentioned in [8], the estimated heading angle will be updated with the heading $\psi_{\mathrm{GNSS}}$ extracted from the GNSS velocity vector when available.

The detection of walking and cycling, based on thresholds on the pitch angle estimation $\theta$, is seamless to the user. Therefore, the proposed algorithm switches automatically between walking and cycling depending on the user's actions. Figure 9 shows the pitch angle estimation of walking and cycling, where walking is highlighted in red and cycling is highlighted in green.

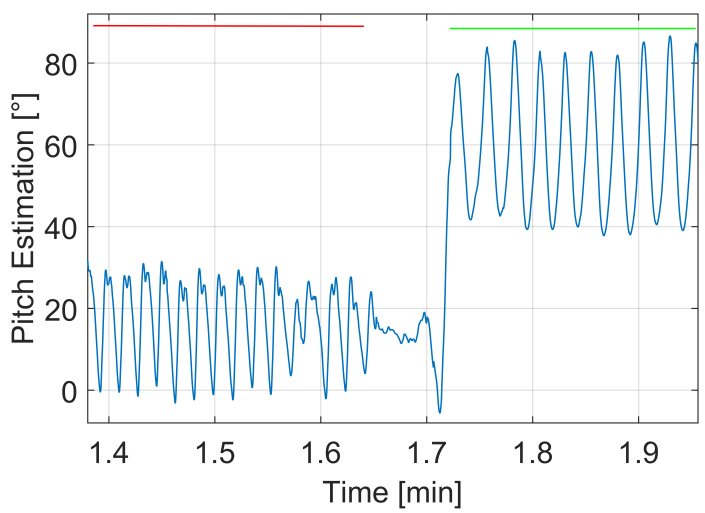

Fig. 9. Pitch angle estimation during walking and cycling periods. Cycling is highlighted in green and walking in red.

The state vector of the position filter is $\boldsymbol{x}^{k}=$ $\left[p_{\mathrm{E}}^{k}, p_{\mathrm{N}}^{k}, p_{\mathrm{U}}^{k}, s^{k}\right]^{T}$, where $\left(p_{\mathrm{E}}, p_{\mathrm{N}}, p_{\mathrm{U}}\right)$ are the East, North and Up components, respectively, of the user's position represented in the East North Up (ENU) navigation frame. The prediction 
stage is defined as follows:

$$
F=\left(\begin{array}{cccc}
1 & 0 & 0 & \Delta t \cdot \cos (\psi) \\
0 & 1 & 0 & \Delta t \cdot \sin (\psi) \\
0 & 0 & 1 & 0 \\
0 & 0 & 0 & 1
\end{array}\right)
$$

If the user is walking, the pitch angle $\theta$ is used to detect steps and compute the displacement estimation $d$, namely step length and vertical displacement (see [9], [10]). Then, the forward speed $s_{\text {INS }}$ derived with the displacement and the time elapsed between steps $\Delta t$, is used as update.

If the user is cycling, the forward speed $\left(s_{\text {INS }}=f_{w} \cdot 2 \pi\right.$. $r)$ is also estimated and applied as update. The frequency of the wheel $f_{w}$ is found when pedaling using the gear $g$ and the pedaling frequency $f_{p}$ and when coasting analyzing the magnetometers' measurements as previously explained.

Independently of the mean of transportation, i.e. walking or cycling, the GNSS measurements are applied to the update stage. Latitude and longitude are converted from the Earth Center Earth Fix (ECEF) frame to ENU navigation frame. Additionally, height as well as forward speed $s_{\mathrm{GNSS}}$ are also used as update.

\section{EXPERIMENTAL RESULTS}

In order to test the above explained ubiquitous and multimodal navigation system, we have carried out a measurement campaign. In the following, we describe the measurement campaign and discuss the performance evaluation of the proposed method.

\section{A. Measurement Campaign}

The measurement campaign has been carried out at the Oberpfaffenhofen airport (Germany). This venue has been selected to have continuous ground truth in an open sky environment (see Figure 10). To that end, we have used a dualfrequency navigation-grade Legacy-E+ GPS/Glonass receiver from Topcon connected to a dual-frequency GNSS antenna fixed onto a helmet worn on the user's head. The recorded raw GNSS measurements have been post-mission processed with Novatel's Grafnav software. The obtained precision with Precise Point Positioning (PPP) processing was around $3 \mathrm{~cm}$ and $6 \mathrm{~cm}$ in East and North direction $(1 \sigma)$, respectively. This highly accurate position has not been integrated in our proposed filter, but used exclusively as a reference to compute the error of our results.

We have tested the means of transportation walking and cycling. For cycling, the activities pedaling, coasting, breaking, speed changes and switching gears were continuously performed.

The volunteers worn a GNSS receiver and inertial and magnetic sensors in four different locations on the body, namely pocket, shoe, wrist and glasses. In this work we will only use the GNSS measurements as well as the accelerometer and turn rate measurements from the pocket-mounted sensor, and the magnetometers' measurements from the shoe-mounted sensors.

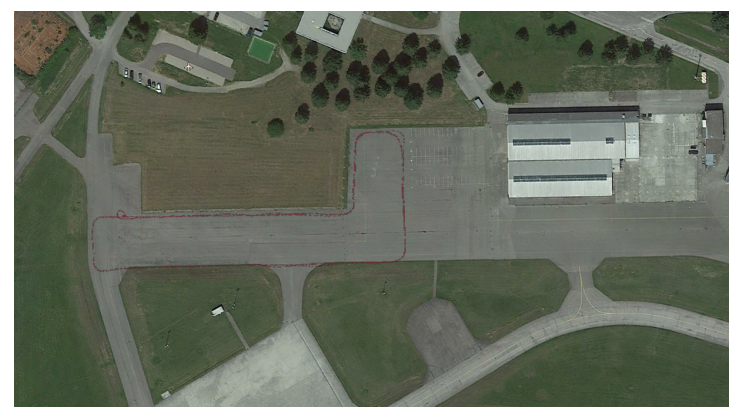

Fig. 10. Bird view of the airport's area where our measurement campaign took place.

The sensors used are the MTw from Xsens, which include inertial and magnetic sensors. In the pocket, though, we have used a MTi sensor, as well from Xsens, because it has also a GNSS receiver embedded. The MTi GNSS receiver was connected to the GNSS antenna on the helmet through a radio frequency splitter. Figure 11 shows the location of the different devices on the test person.

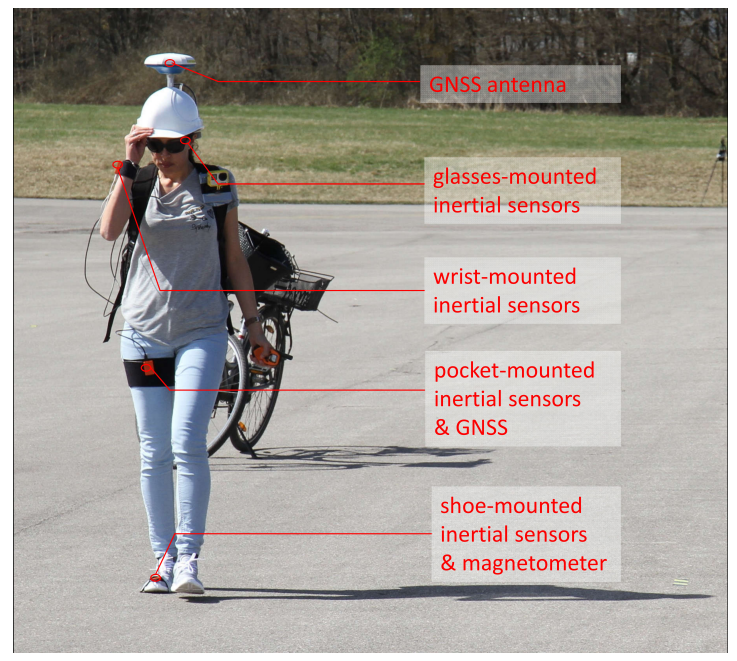

Fig. 11. This picture highlights the sensor's placement on the volunteers during the measurement campaign.

Both, inertial and magnetic measurements are recorded at a rate of $100 \mathrm{~Hz}$. The GNSS solution used in our proposed filter is outputted by the proprietary software from Xsens at a rate of $4 \mathrm{~Hz}$. We have recorded all data using this proprietary software running in a notebook stored in the backpack of the user. All mentioned sensors are connected via cable to the notebook and the provided measurements are internally synchronized.

We have recorded data from three different bicycles and three different volunteers. In total, we own a more than $12 \mathrm{~km}$ length data base that is available under [13].

\section{B. Evaluation}

In this section we present and analyze the results of the aforementioned measurement campaign. First, we test the proposed coasting algorithm using only inertial sensors mounted in the pocket and the magnetometer mounted in the shoe. 
Second, we show the performance of our proposed multimodal intelligent urban navigation system based on the loose fusion INS/GNSS and magnetometers.

1) Coasting for Bicycle Navigation: As previously explained, the coasting is still an open issue for bicycle navigation systems in GNSS denied scenarios and without using vehicle-mounted sensors.

In the following, we show two examples of rides including pedaling, braking and coasting. Braking and coasting are not differentiated, since both are treated using the proposed algorithm.

Figure 12 shows two repetitions of the proposed trajectory for one of the volunteers. The experiment is performed entirely riding a bicycle and starts at $(0,0)$. All detected coasting periods are highlighted in red. As expected, coasting occurs naturally in long straight parts and when approaching a turn.

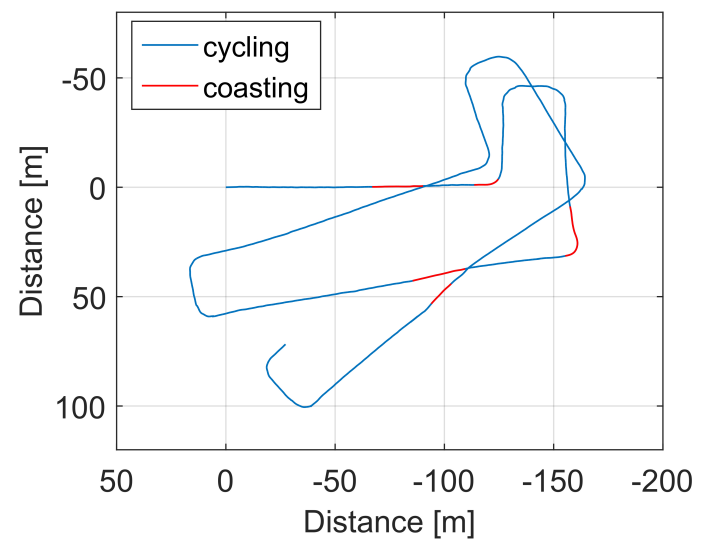

Fig. 12. The blue curve shows the trajectory described for this experiment, while the coasting periods are highlighted in red.

The heading angle is estimated by the INS mounted in the pocket. The accumulated drift that can be appreciated in the figure, arises from the lack of corrections from GNSS measurements, which are not used for this experiment.

Table I summarizes the results for this experiment. The distance estimated while coasting is in total $99 \mathrm{~m}$. During these periods, the proposed coasting algorithm has been used to derive the forward speed of the bicycle. The computed travelled distance during both, pedaling and coasting, differs only $1.2 \%$ from the ground truth travelled distance extracted from the ground truth system. Without using the proposed algorithm, the coasting periods would have been computed as zero forward speed, since the pedaling frequency is zero.

TABLE I

VALUES FOR VOLUNTEER 1

\begin{tabular}{|l|c|}
\hline Total travelled distance & $940 \mathrm{~m}$ \\
\hline Detected \& estimated distance coasting & $99 \mathrm{~m}$ \\
\hline Distance error without proposed coasting algorithm & $10.7 \%$ \\
\hline Distance error with proposed coasting algorithm & $1.2 \%$ \\
\hline
\end{tabular}

Figure 13 shows the same experiment performed by a different volunteer. The detected coasting periods are highlighted in red. In this case, the obtained results are very similar to the previous realization.

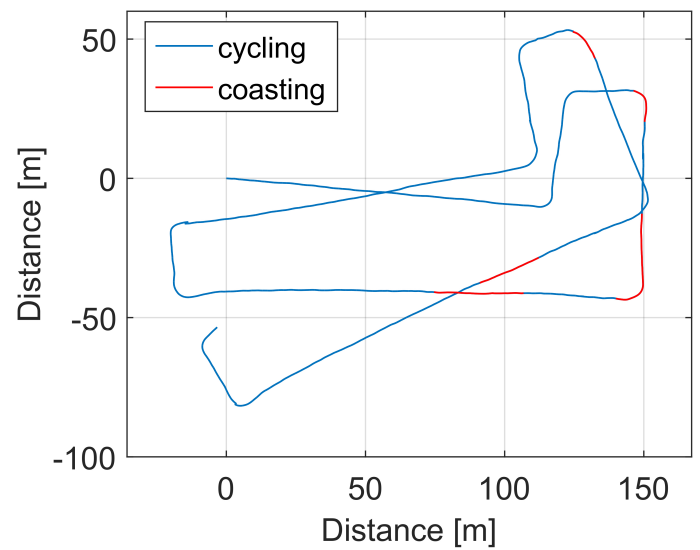

Fig. 13. The blue curve shows the trajectory described for this experiment, while the coasting periods are highlighted in red.

The total estimated travelled distance while cycling and coasting differs $2 \%$ from the ground truth travelled distance measured by the ground truth system. The results are summarized in Table II.

TABLE II

VALUES FOR VOLUNTEER 2

\begin{tabular}{|l|c|}
\hline Total travelled distance & $940 \mathrm{~m}$ \\
\hline Detected \& estimated distance coasting & $140 \mathrm{~m}$ \\
\hline Distance error without proposed coasting algorithm & $17 \%$ \\
\hline Distance error with proposed coasting algorithm & $2 \%$ \\
\hline
\end{tabular}

We conclude that our proposed method based on the pitch angle is able to seamlessly detect coasting or braking periods during a cycling ride. Further than detecting, it also computes the forward speed of the bicycle, or the travelled distance, using magnetometers' measurements of the sensor mounted in the shoe providing errors below $2 \%$ of the true travelled distance for approximately $15 \%$ of coasting during the ride.

2) Multimodal Intelligent Urban Navigation: In this section we test the performance of our proposed multimodal intelligent urban navigation system. Therefore, experiments including walking and multi-gear cycling will be analyzed. The targeted GNSS-denied scenarios, e.g. urban tunnel, will be simulated by disconnecting the GNSS updates.

Figure 14 shows seven repetitions of the proposed trajectory for one of the volunteers. Unlike the previous section, these results are estimated using the loose fusion INS/GNSS, thus the heading angle is corrected using GNSS measurements. The coasting periods are highlighted in red. In this case, a GNSS outage of $20 \mathrm{~s}$ has been simulated when the volunteer was riding on a straight path. The outage starts approximately at $(-10,50)$.

Table III summarizes the results for this experiment. The distance estimated during the $20 \mathrm{~s}$ GNSS outage is $73 \mathrm{~m}$. The absence of GNSS measurements has been bridged with the proposed INS and magnetometer based algorithm obtaining 


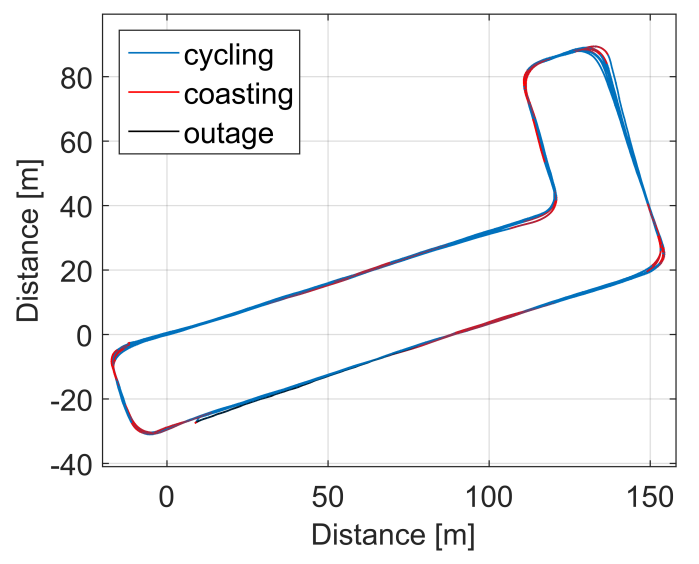

Fig. 14. The blue curve shows the trajectory described for this experiment. The coasting periods are highlighted in red and the path travelled during the GNSS outage is shown in black.

a position error of $2.5 \mathrm{~m}$. This error has been computed as the straight line joining the last estimated position during the GNSS outage and the first corrected position.

TABLE III

VALUES FOR 20 s OUTAGE

\begin{tabular}{|l|c|}
\hline Total travelled distance & $2857 \mathrm{~m}$ \\
\hline Estimated distance travelled during outage & $73 \mathrm{~m}$ \\
\hline Position error with proposed intelligent algorithm & $2.5 \mathrm{~m}$ \\
\hline
\end{tabular}

Figure 15 shows the same experiment. The GNSS outage, though, has been simulated over $40 \mathrm{~s}$ during a curved part of the trajectory. The coasting periods are highlighted in red and the trajectory travelled during the GNSS outage is shown in black. The outage starts approximately at $(-5,75)$.

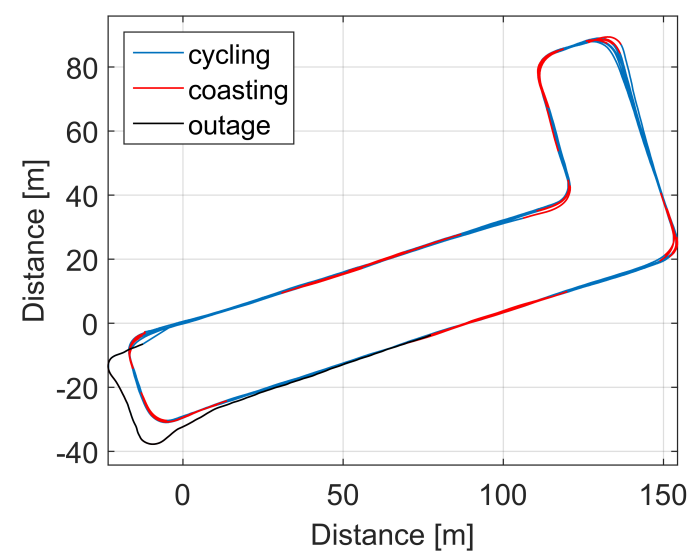

Fig. 15. The blue curve shows the trajectory described for this experiment. The coasting periods are highlighted in red and the path travelled during the GNSS outage is shown in black.

Table IV summarizes the results for this experiment. In this case the position error accumulated during the GNSS outage rises up to $9.5 \mathrm{~m}$. The period during the GNSS outage is mostly covered coasting and braking, because it occurs in two consecutive curves. Therefore, it is more challenging than a pedaling situation on a straight trajectory and, additionally, the outage is twice as long as the previous GNSS outage.

TABLE IV

VALUES FOR 40 s OUTAGE

\begin{tabular}{|l|c|}
\hline Total travelled distance & $2857 \mathrm{~m}$ \\
\hline Estimated distance travelled during outage & $134 \mathrm{~m}$ \\
\hline Position error with proposed intelligent algorithm & $9.5 \mathrm{~m}$ \\
\hline
\end{tabular}

We conclude that the proposed algorithm successfully bridges the distance between GNSS fixes including changes of speed and different activities such as pedaling, braking and coasting.

For the next experiment, different means of transportation, e.g. walking and cycling, will be tested. This experiment shown in Figure 16, also covers seven repetitions of the proposed trajectory, where the last $320 \mathrm{~m}$ are performed walking. The GNSS outage of $40 \mathrm{~s}$ duration is simulated when the user walks. The figure does not highlight the outage for clarity.

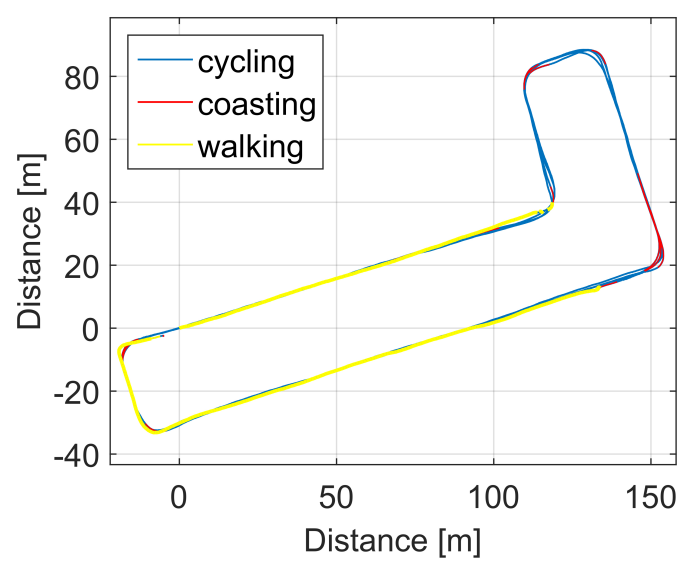

Fig. 16. The blue curve shows the trajectory described for this experiment The coasting periods are highlighted in red and the path covered walking is shown in yellow.

Table V summarizes the results for this experiment. In this case the position error accumulated during the GNSS outage is $1 \mathrm{~m}$. Since the outage has been simulated during walking, the travelled distance is estimated using the INS mounted in the pocket. The accumulated error is due to the heading estimation that was not updated with GNSS measurements over $56 \mathrm{~m}$.

TABLE V

VALUES FOR 40 s OUTAGE WALKING

\begin{tabular}{|l|c|}
\hline Total travelled distance & $2857 \mathrm{~m}$ \\
\hline Estimated distance travelled during outage & $56 \mathrm{~m}$ \\
\hline Estimated distance travelled walking & $320 \mathrm{~m}$ \\
\hline Position error with proposed intelligent algorithm & $1 \mathrm{~m}$ \\
\hline
\end{tabular}

We conclude that the proposed intelligent multimodal navigation system for urban environments is able to cope with the modes of transportation walking and riding a bicycle and 
successfully bridges short periods of GNSS measurements absence or periods where the quality of the GNSS measurements makes them not to be taken into account.

\section{Conclusions And OUtLOOK}

We proposed a multimodal intelligent navigation system for urban environments based on wearable sensors. We also proposed a novel algorithm to compute the forward speed of the bicycle during coasting and braking periods in GNSSdenied scenarios.

We have performed an extensive measurement campaign and we have used post-processed GNSS Precise Point Positioning (PPP) as reference system to compute the error. We prove that our method is able to successfully estimate the forward speed of the bicycle during coasting or braking periods. Likewise, we prove that our navigation system switches seamlessly between walking and riding a bicycle and is also able to bridge short periods of absence of GNSS measurements.

Our next step will consist of integrating the GNSS and INS measurements in a tightly fashion and include other means of transportation.

\section{REFERENCES}

[1] C. Premebida, O. Ludwig, and U. Nunes, "LIDAR and Vision-based Pedestrian Detection System," J. Field Robotics, vol. 26, pp. 696-711, 2009.

[2] R. Benenson, M. Omran, J. Hosang, and S. B., "Ten Years of Pedestrian Detection, What Have We Learned?" Agapito L., Bronstein M., Rother C. (eds) Computer Vision- ECCV 2014 Workshops. ECCV 2014. Lecture Notes in Computer Science, vol. 8926, 2015.

[3] E. Munoz Diaz, A. L. Mendiguchia Gonzalez, and F. de Ponte Müller, "Standalone Inertial Pocket Navigation System," IEEE/ION Position Location and Navigation Symposium (PLANS), 2014.

[4] Z. Xiao, H. Wen, A. Markham, and N. Trigoni, "Robust Pedestrian Dead Reckoning (R-PDR) for Arbitrary Mobile Device Placement," IEEE International Conference on Indoor Positioning and Indoor Navigation (IPIN), 2014.

[5] J. W. Kim, H. J. Jang, D.-H. Hwang, and C. Park, "A Step, Stride and Heading Determination for the Pedestrian Navigation System," Journal of Global Positioning Systems, vol. 3, no. 1-2, pp. 273-279, 2004.

[6] V. Renaudin, M. Susi, and G. Lachapelle, "Step Length Estimation Using Handheld Inertial Sensors," Sensors, vol. 12, no. 7, pp. 8507-8525, 2012.

[7] V. Renaudin, V. Demeule, and M. Ortiz, "Adaptative Pedestrian Displacement Estimation with a Smartphone," IEEE International Conference on Indoor Positioning and Indoor Navigation (IPIN), vol. 12, pp. 916-924, 2013.

[8] Geographical and Fingerprinting Data for Positioning and Navigation Systems. Section 8: Other Technologies to Position and Navigate in Indoor Environments. Elsevier, 2018.

[9] E. Munoz Diaz, "Inertial Pocket Navigation System: Unaided 3D Positioning," Journal of Sensors, vol. 15, pp. 9156-9178, 2015.

[10] E. Munoz Diaz and A. L. Mendiguchia Gonzalez, "Step Detector and Step Length Estimator for an Inertial Pocket Navigation System," IEEE International Conference on Indoor Positioning and Indoor Navigation (IPIN), 2014.

[11] H.-W. K. Chang, N. El-Sheimy, and J. Georgy, "Cycling Derived Models for Enhancing Navigation Performance of a Low Cost Multi-Sensors System," Journal of Aeronautics, Astronautics and Aviation, Series A, vol. 5, pp. 179-186, 2013.

[12] H.-W. K. Chang, J. Georgy, and N. El-Sheimy, "Cycling Dead Reckoning for Enhanced Portable Device Navigation on Multi-gear Bicycles," GPS Solutions, vol. 19, p. 611621, 2015.

[13] "Data base of the raw measurements evaluated in this article," www.ftp.dlr.de; User name:PDR_Walks_LargeAreas_guest; Password:xU4nVwuz; Ports: 20-21. 